\title{
On admissible curves and their evolution equations in pseudo-Galilean space
}

\author{
Hossam S. Abdel-Aziz ${ }^{a}, *$, Hebatallah M. Serry ${ }^{b, *}$, Fifi M. El-Adawy ${ }^{b}$, Amal A. Khalil ${ }^{a}$ \\ ${ }^{a}$ Department of Mathematics, Sohag University, Sohag 82524, Egypt. \\ ${ }^{b}$ Department of Mathematics, Suez Canal University, Ismailia, Egypt.
}

\begin{abstract}
The evolution equations of some forms of admissible curves in the pseudo-Galilean Space $\mathrm{G}_{3}^{1}$ are investigated in this paper. In more detail, we use two separate methods to obtain coupled nonlinear partial differential equations of time evolution in terms of their curvatures. The first method studies the evolution equations for admissible curves via the frame field, while the second studies the evolution equations via the velocity vector. Then, the position vectors of the evolving curves are formulated. Also, we conduct comparative research of the evolution equations for curves in different spaces. We furthermore present some models as an application of the evolution equations of the curvature and torsion for admissible curves, confirming our theoretical results.
\end{abstract}

Keywords: Admissible curves, evolution equations, frenet frame, pseudo-Galilean space, spacelike and timelike curves.

2020 MSC: 53A35, 53E99.

(c)2022 All rights reserved.

\section{Introduction}

In differential geometry, despite existing tons of studies regarding curves and surfaces and the way they evolve per time, recent studies have created a massive improvement in the evolution of the curves and surfaces. Studying evolution equations for curves and surfaces from a geometrical perspective is a core research topic in the field of differential geometry. Evolution equations have a wide range of applications in a wide range of fields, attracting the attention of a wide range of scholars, regardless of their theoretical or implementation backgrounds. They usually arise from multiple applications in many engineering and physical applications such as material science, dynamics of section boundaries in thermomechanics, computational geometry, semiconductor industry, image processing, mathematical modelling, scientific computing, and computer vision. Also, they can be investigated in a purely mathematical context $[9,17$, $19,25,31-34]$. In physics, evolution equations of curves have many critical applications such as magnetic spin chains and vortex filaments [5, 16, 21, 29]. Obtaining the evolution equations and learning how the curves evolve over time is an exciting and difficult problem that has been reviewed in a variety of spaces by several research studies, [1, 2, 18, 24]. Another related work is [3], in which Abdel-All et al. took a novel approach to the time evolution of a curve by obtaining the evolution equations of a generalized space curve. Then, Yild1z et al. studied the evolution of the generalized space curve in Minkowski Space, [35]. Several scholars have recently studied geometric flow problems for curves, [4, 20].

\footnotetext{
*Corresponding author

Email addresses: habdelaziz2005@yahoo.com (Hossam S. Abdel-Aziz), hebamserry@gmail.com (Hebatallah M. Serry), fmorsi88@yahoo.com (Fifi M. El-Adawy), amalaboelwafa@yahoo.com (Amal A. Khalil)
}

doi: $10.22436 /$ jmcs.025.04.07 
In our work, the evolution of curves via the velocities of their moving frame in pseudo-Galilean space $\mathrm{G}_{3}^{1}$ has been studied. Then, we acquire a combination of coupled nonlinear partial differential equations that govern the time evolution of the curvatures for the meant curves in pseudo-Galilean space $\mathrm{G}_{3}^{1}$. The solution of the coupled nonlinear partial differential equations was found by the Mathematica software system employing a package that applies tanh-sech methods [6]. To obtain curves from their curvatures we expand the Frenet equations numerically up to their position $[2,6]$. Then curves and their position vectors that correspond to those solutions are shown.

The following is how the rest of the paper is organized. Section 1 contains an introduction to the work and the aim of this paper. In Section 2, we offer a short outline of the fundamentals and preliminaries for the geometry of curves in pseudo-Galilean space $\mathrm{G}_{3}^{1}$. The evolution equations of admissible curves and how to obtain them have been studied using their frame field in Section 3 and using their velocity vector in Section 4. In Section 5, we compare the evolution equations for different spaces. Finally, Section 6 concludes the paper.

\section{Preliminaries on pseudo-Galilean geometry}

This section covers the fundamental concepts as well as the theory of curves in pseudo-Galilean space $\mathrm{G}_{3}^{1}$; for more details, see $[11,12]$. The pseudo-Galilean geometry is one of the core geometries of CayleyKlein with a signature metric of $(0,0,+,-)$. The pseudo-Galilean space $\mathrm{G}_{3}^{1}$ is a three-dimensional projective space in which the absolute figure is an ordered triple $(\sigma, g, M)$, where $\sigma$ is the absolute plane, $g$ is the absolute line in $\sigma$ and $M$ is the fixed hyperbolic spin of points of $g$ [8]. If a plane contains $g$, it is called a pseudo-Euclidean plane; otherwise, it is called isotropic.The planes $\Pi=$ const, as well as the planes $\sigma$, are pseudo-Euclidean planes. The rest of the planes are isotropic. by

In pseudo-Galilean $G_{3}^{1}$, the inner product of two vectors $a=\left(a_{1}, a_{2}, a_{3}\right)$ and $b=\left(b_{1}, b_{2}, b_{3}\right)$ is outlined

$$
\langle a, b\rangle_{G_{3}^{1}}= \begin{cases}a_{1} b_{1}, & \text { if } a_{1} \neq 0 \text { or } b_{1} \neq 0 \\ a_{2} b_{2}-a_{3} b_{3}, & \text { if } a_{1}=0 \text { and } b_{1}=0 .\end{cases}
$$

If $u_{1} \neq 0$, a vector $u=\left(u_{1}, u_{2}, u_{3}\right)$ is said to be non-isotropic. As a consequence, all unit non-isotropic vectors have the form $u=\left(1, u_{2}, u_{3}\right)$. For isotropic vectors, $\mathfrak{u}_{1}=0$ holds true. There are four major kinds of isotropic vectors; spacelike $\left(u_{1}=0, u_{2}^{2}-u_{3}^{2}>0\right)$, timelike $\left(u_{1}=0, u_{2}^{2}-u_{3}^{2}<0\right)$ and two kinds of lightlike vectors if $\left(u_{1}=0, u_{2}= \pm u_{3}\right)$. A non-lightlike isotropic vector is a unit vector if $u_{2}^{2}-u_{3}^{2}= \pm 1$ [11, 12]. See [13-15] for more information. The orthogonality of the vectors $a=\left(0, a_{2}, a_{3}\right)$ and $b=\left(0, b_{2}, b_{3}\right)$, i.e., $a \perp b$ means $a b=0$. The pseudo-Galilean vector product of two vectors $a=\left(a_{1}, a_{2}, a_{3}\right)$ and $b=\left(b_{1}, b_{2}, b_{3}\right)$ is defined as

$$
a \wedge_{G_{3}^{1}} b=\left|\begin{array}{ccc}
0 & -e_{2} & e_{3} \\
a_{1} & a_{2} & a_{3} \\
b_{1} & b_{2} & b_{3}
\end{array}\right|=\left(0, a_{3} b_{1}-a_{1} b_{3}, a_{2} b_{1}-a_{1} b_{2}\right),
$$

where $e_{2}=(0,1,0)$ and $e_{3}=(0,0,1)$.

In pseudo-Galilean space $G_{3}^{1}$, a curve $\Gamma$ is defined as $\Gamma: I \rightarrow G_{3}^{1}, \Gamma(t)=(\zeta(t), \vartheta(t), \rho(t))$, where $I \subset R$ and $\zeta(t), \vartheta(t), \rho(t) \in C^{3}$. A given curve $\Gamma(t)$ is said to be admissible, if $\zeta \cdot(t) \neq 0[11,12]$. Any admissible curve has the form $\Gamma(\zeta)=(\zeta, \vartheta(\zeta), \rho(\zeta))$, which is referred to as an admissible curve of the first type. For simplicity, we assume that $d s=d \zeta$ and $s=\zeta$ as the arc-length parameter of $\Gamma$. The following two forms of admissible curves are discussed using the absolute figure of pseudo-Galilean space $\mathrm{G}_{3}^{1}$.

\subsection{The admissible curves of the first type}

Allow $\Gamma: I \rightarrow G_{3}^{1}$, I $\subset \mathrm{R}$ be an admissible curve parametrized by the pseudo-Galilean invariant parameter $s=\zeta$, as mentioned previously. Then Frenet formulas are as follows $[7,12,13]$

$$
\left(\begin{array}{l}
\mathbf{T}(\zeta) \\
\mathbf{n}(\zeta) \\
\mathbf{p}(\zeta)
\end{array}\right)_{\zeta}=\left(\begin{array}{ccc}
0 & \kappa(\zeta) & 0 \\
0 & 0 & \tau(\zeta) \\
0 & \tau(\zeta) & 0
\end{array}\right)\left(\begin{array}{l}
\mathbf{T}(\zeta) \\
\mathbf{n}(\zeta) \\
\mathbf{p}(\zeta)
\end{array}\right)
$$


where,

$$
\left\{\begin{array}{l}
\mathbf{T}(\zeta)=\Gamma_{\zeta}=\left(1, \vartheta^{\prime}(\zeta), \rho^{\prime}(\zeta)\right) \\
\mathbf{n}(\zeta)=\frac{1}{\kappa}\left(0, \vartheta^{\prime \prime}(\zeta), \rho^{\prime \prime}(\zeta)\right) \\
\mathbf{p}(\zeta)=\frac{1}{\kappa}\left(0, \epsilon \rho^{\prime \prime}(\zeta), \epsilon \vartheta^{\prime \prime}(\zeta)\right)
\end{array}\right.
$$

are the vectors of the tangent, the central isotropic normal (simply it is known as principal normal or normal), and the binormal line of $\Gamma(\zeta)$, respectively. The functions $\kappa(\zeta)=\sqrt{\left|\vartheta^{\prime \prime}(\zeta)^{2}-\rho^{\prime \prime}(\zeta)^{2}\right|}$ and $\tau(\zeta)=$ $\frac{1}{\kappa^{2}} \operatorname{det}\left[\Gamma^{\prime}, \Gamma^{\prime \prime}, \Gamma^{\prime \prime \prime}\right]$ are geometric parameters that represent, respectively, the curvature and torsion of $\Gamma(\zeta)$ and $\epsilon= \pm 1$, are chosen by criterion det $[\mathbf{T}, \mathbf{n}, \mathbf{p}]=1$. Through this paper, the subscripts describe partial derivatives. An admissible curve $\Gamma(\zeta)$ is timelike (resp. spacelike) if the principal normal $\mathbf{n}$ is a spacelike (resp. timelike) vector. The normal vectors spacelike when they are $\epsilon=+1$ and timelike whenever they are $\epsilon=-1[7,12]$. As known, when the Frenet frame $\{\mathbf{T}, \mathbf{n}, \mathbf{p}\}$ moves along an admissible curve $\Gamma$ in pseudo-Galilean space $G_{3}^{1}$, the shear motion is determined by an angular velocity vector (Darboux vector), which has the equation

$$
\mathbf{W}(\zeta)=\epsilon(\tau(\zeta) \mathbf{T}(\zeta)-\kappa(\zeta) \mathbf{p}(\zeta))
$$

where $\epsilon= \pm 1$ depending on the normal type as mentioned before. The Darboux vector satisfies Darboux equations which are given by

$$
\frac{\mathrm{d} \mathbf{T}}{\mathrm{d} \zeta}=\mathbf{W}(\zeta) \wedge \mathbf{T}, \quad \frac{\mathrm{d} \mathbf{n}}{\mathrm{d} \zeta}=\mathbf{W}(\zeta) \wedge \mathbf{n}, \quad \frac{\mathrm{d} \mathbf{p}}{\mathrm{d} \zeta}=\mathbf{W}(\zeta) \wedge \mathbf{p}
$$

The related trihedron's Frenet vector is often split into two shear motions: $\mathbf{p}$ binormal vector shear with $-\epsilon K$ angular speed along absolutely the line, that is

$$
\frac{\mathrm{d} \mathbf{T}}{\mathrm{d} \zeta}=(-\epsilon \kappa \mathbf{p}) \wedge \mathbf{T}
$$

and $\mathbf{n}$ normal vector shear with $\epsilon \tau$ angular speed along the absolute line, that is

$$
\frac{\mathrm{d} \mathbf{n}}{\mathrm{d} \zeta}=(\epsilon \tau \mathbf{T}) \wedge \mathbf{n}
$$

\subsection{The admissible curves of the second type}

In this case, an admissible curve $\alpha$ are often written as

$$
\alpha(\zeta)=(0, \vartheta(\zeta), \rho(\zeta)) .
$$

Then the Frenet formulae of $\alpha(\zeta)$ are given as in (2.1) with tangent, normal, and the binormal lines, respectively given by,

$$
\left\{\begin{array}{l}
\mathbf{T}(\zeta)=\left(1, a_{2}(\zeta), a_{3}(\zeta)\right), \\
\mathbf{n}(\zeta)=\left(0, \rho^{\prime}(\zeta), \vartheta^{\prime}(\zeta)\right) \\
\mathbf{p}(\zeta)=\left(0, \vartheta^{\prime}(\zeta), \rho^{\prime}(\zeta)\right)
\end{array}\right.
$$

where $\mathbf{a}(\zeta)=\left(1, \mathrm{a}_{2}(\zeta), \mathrm{a}_{3}(\zeta)\right)$ is a unit vector field. The curvature and the torsion of $\alpha$ are, respectively,

$$
\kappa(\zeta)=-\frac{a_{2}}{\rho^{\prime}}, \quad \tau(\zeta)=\frac{\vartheta^{\prime \prime}}{\rho^{\prime}} .
$$

Also, we have the same Darboux vector as given in (2.2) which satisfies Darboux equations (2.3) too.

\section{Evolution equations of admissible curves via their frame fields in pseudo-Galilean space}

In pseudo-Galilean space $G_{3}^{1}$, let $\Gamma(\zeta)=(\zeta, \vartheta(\zeta), \rho(\zeta))$ be an admissible curve of the first type. Then the associated trihedron (Eq. (2.1)) of the curve $\Gamma$ can then be represented in a matrix form as

$$
\frac{\partial \mathbf{E}}{\partial \zeta}=\mathbf{A} \mathbf{E},
$$


where,

$$
\mathbf{E}=\left(\begin{array}{c}
\mathbf{T}(\zeta) \\
\mathbf{n}(\zeta) \\
\mathbf{p}(\zeta)
\end{array}\right), \quad \mathbf{A}=\left(\begin{array}{ccc}
0 & \kappa(\zeta) & 0 \\
0 & 0 & \tau(\zeta) \\
0 & \tau(\zeta) & 0
\end{array}\right)
$$

If $\Gamma(\zeta)$ moves with time $t$; then the trihedron $\{\mathbf{T}, \mathbf{n}, \mathbf{p}\}$ and the curvatures become functions of both $\zeta$ and $t$, we can compute the time evolution of $\mathbf{T}, \mathbf{n}$, and $\mathbf{p}$ to get

$$
\frac{\partial \mathbf{E}}{\partial \mathrm{t}}=\mathbf{F} \mathbf{E}
$$

with noting that

$$
\mathbf{E}=\left(\begin{array}{c}
\mathbf{T}(\zeta, \mathrm{t}) \\
\mathbf{n}(\zeta, \mathrm{t}) \\
\mathbf{p}(\zeta, \mathrm{t})
\end{array}\right), \mathbf{F}=\left(\begin{array}{ccc}
0 & \epsilon \Omega_{3}(\zeta, t) & \epsilon \Omega_{2}(\zeta, t) \\
0 & 0 & -\epsilon \Omega_{1}(\zeta, t) \\
0 & -\epsilon \Omega_{1}(\zeta, t) & 0
\end{array}\right) .
$$

The motion of the curve $\Gamma$ is detected by $\Omega_{1}, \Omega_{2}$, and $\Omega_{3}$ (which are the velocities of the moving frame). One of the main results in this paper can be stated as in the following Theorem 3.1.

Theorem 3.1 (Equations of motion for an admissible curve of first type). Let $\Gamma$ be an admissible curve of the first type in pesudo-Galilean space $\mathrm{G}_{3}^{1}$. For $\Gamma$ curve, the compatibility condition is satisfied between (3.1) and (3.2), i.e.,

$$
\frac{\partial}{\partial t} \frac{\partial E}{\partial \zeta}=\frac{\partial}{\partial \zeta} \frac{\partial E}{\partial t}
$$

and we get a system of coupled non linear partial differential equations that describe the motion equations of the curve via its frame field $\{\boldsymbol{T}, \boldsymbol{n}, \boldsymbol{p}\}$

$$
\left\{\begin{array}{l}
\kappa_{\mathrm{t}}=\epsilon\left(-\left(\frac{\kappa \Omega_{1}+\Omega_{2 \zeta}}{\tau}\right) \zeta+\tau \Omega_{2}\right), \\
\tau_{\mathrm{t}}=-\epsilon \Omega_{1 \zeta}
\end{array}\right.
$$

Proof. Using the compatibility condition (eq (3.3)), which after some calculations yields the following equation:

$$
\frac{\partial \mathbf{A}}{\partial t}-\frac{\partial \mathbf{F}}{\partial \zeta}+[\mathbf{A}, \mathbf{F}]=0_{3 \times 3}
$$

where,

$$
[\mathbf{A}, \mathbf{F}]=\mathbf{A F}-\mathbf{F A}
$$

is Lie bracket of $\mathbf{F}$ and $\mathbf{A}$. In view of the above, Eq. (3.5) reads as follows:

$$
\left(\begin{array}{ccc}
0 & \kappa_{\mathrm{t}}-\epsilon\left(\Omega_{3 \zeta}+\tau \Omega_{2}\right) & -\epsilon\left(\kappa \Omega_{1}+\Omega_{2 \zeta}+\tau \Omega_{3}\right) \\
0 & 0 & \tau_{\mathrm{t}}+\epsilon \Omega_{1 \zeta} \\
0 & \tau_{\mathrm{t}}+\epsilon \Omega_{1 \zeta} & 0
\end{array}\right)=0_{3 \times 3},
$$

that gives

$$
\left\{\begin{array}{l}
\kappa_{\mathrm{t}}=\epsilon\left(\Omega_{3 \zeta}+\tau \Omega_{2}\right) \\
\Omega_{3}=-\left(\frac{\Omega_{2 \zeta}+\kappa \Omega_{1}}{\tau}\right) \\
\epsilon \Omega_{1 \zeta}=-\tau_{\mathrm{t}}
\end{array}\right.
$$

which can be reduced to a set of coupled nonlinear partial differential equations in the form of (3.4).

By dissolving the system (3.4) with the Mathematica software program [30], we can get the equations of motion of the admissible curve $\Gamma$ for a given $\Omega_{1}, \Omega_{2}, \Omega_{3}$ and the curvatures of the admissible curve through numerical integration of Frenet equations (1). After choosing $\left\{\Omega_{1}, \Omega_{2}, \Omega_{3}\right\}$ in terms of the $\{K, \tau\}$ and their derivatives, the parameters $\left\{k, \tau, \Omega_{1}, \Omega_{2}, \Omega_{3}\right\}$ are a description of the moving admissible curve, and the solutions generate the evolution of the admissible curve with time $t$. 
If $\left\{\Omega_{1}, \Omega_{2}, \Omega_{3}\right\}=\{\tau, 0, \kappa\}$, then the system (3.7) above takes the form:

$$
\left\{\begin{array}{l}
\kappa_{\mathrm{t}}=-\epsilon \kappa_{\zeta}, \\
\tau_{\mathrm{t}}=-\epsilon \tau_{\zeta},
\end{array}\right.
$$

which has the solution

$$
\kappa=f(\zeta-\epsilon t), \tau=g(\zeta-\epsilon t),
$$

where $f$ and $g$ are arbitrary functions of $\zeta$, t.

The following sections some alternative models for specific curvature and torsion values, as well as their representations in the pseudo-Galilean space $\mathrm{G}_{3}^{1}$.

First Model. Let $\Gamma$ be a spacelike admissible curve with timelike normal. The curvature and torsion are, respectively $\mathrm{K}=$ constant $=-1, \tau=$ constant $=1$, then the position vector of $\Gamma$ at $t=0$ is [28]:

$$
\mathbf{r}(\zeta)=\left(\zeta, \int\left(\int[-\cosh (\zeta+A)] d \zeta\right) d \zeta, \int\left(\int[-\sinh (\zeta+A)] d \zeta\right) d \zeta\right),
$$

as well as,

$$
\mathbf{r}(\zeta)=(\zeta,-\cosh (\zeta+A)+B \zeta+D,-\sinh (\zeta+A)+C \zeta+F),
$$

integration constants are A, B, C, D, and F. Also, the representation of the evolves curve can be seen in Figure 1.

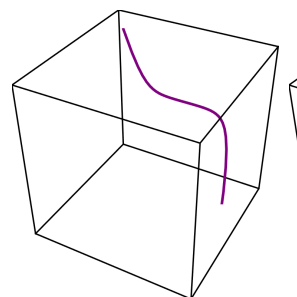

(a) $t=-1$

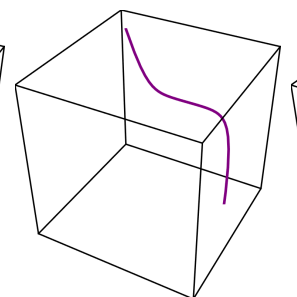

(b) $t=0$

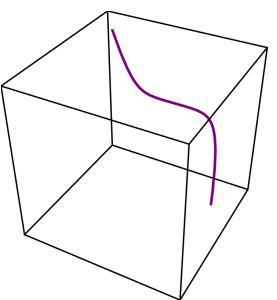

(c) $t=1$

Figure 1: With curve length $20(-10<\zeta<10)$.

If we set $A=B=C=D=F=0$, we obtain the position vector as

$$
\mathbf{r}(\zeta)=(\zeta,-\cosh (\zeta),-\sinh (\zeta)),
$$

which has the shape shown in Figure 2 in pseudo-Galilean space $G_{3}^{1}$.

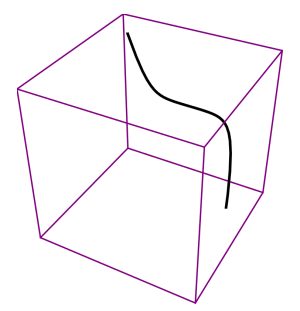

Figure 2: With curve length $20(-10<\zeta<10)$.

We get the numerical representation of the curve (as in Figure 1 at $t=0$ ) is identical to the representation of the curve from its position vector as in Figure 2. 
Second Model. For a given $\Gamma$ as above, take $k=\cos (\zeta+t), \tau=2$. The position vector of the curve is then given relative to its frame from [28]:

$$
\mathbf{r}(\zeta)=\left(\zeta, \int\left(\int\left[\cos (\zeta) \cosh \left(\int 2 \mathrm{~d} \zeta\right)\right] \mathrm{d} \zeta\right) \mathrm{d} \zeta, \int\left(\int\left[\cos (\zeta) \sinh \left(\int 2 \mathrm{~d} \zeta\right)\right] \mathrm{d} \zeta\right) \mathrm{d} \zeta\right),
$$

which yields,

$$
\mathbf{r}(\zeta)=(\zeta, A(\sin \zeta \sinh \zeta)+B \zeta+C, A(\cosh \zeta \sin \zeta)+D \zeta+F),
$$

the integration constants $A, B, C$, and $D$ are used. And, the admissible curve $\Gamma$ is represented by Figure 3. Let's say $A=1$ and $B=C=D=F=0$, the position vector will be as follows:

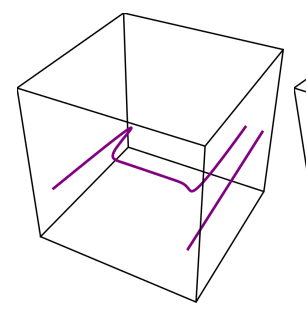

(a) $t=-1$

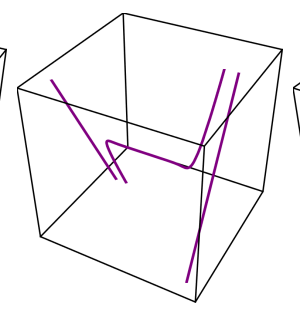

(b) $t=0$

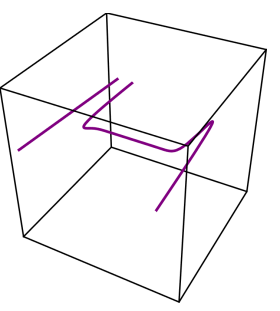

(c) $t=1$

Figure 3: With curve length $20(-10<\zeta<10)$.

$$
\mathbf{r}(\zeta)=(\zeta, \sin \zeta \sinh \zeta, \cosh \zeta \sin \zeta),
$$

which has the representation as in Figure 4.

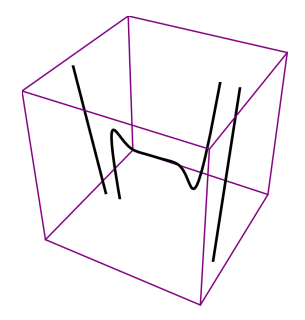

Figure 4: With curve length $20(-10<\zeta<10)$.

It's worth noting that the numerical (Figure $3 \mathrm{~b}$ ) and exact (Figure 4) representations of the curve vary slightly.

Third Model. Similarly, Consider $\Gamma$ be a timelike admissible curve with spacelike normal. The curvature and the torsion of $\Gamma$ are, respectively $k=\cos (\zeta-t), \tau=\sin (\zeta-t)$. When $t=0$, the position vector of the curve with respect to its standard frame can be represented as [28],

$$
\mathbf{r}(\zeta)=\left(\zeta, \int\left(\int\left[\cos (\zeta) \cosh \left(\int[\sin (\zeta)] \mathrm{d} \zeta\right)\right] \mathrm{d} \zeta\right) \mathrm{d} \zeta, \int\left(\int\left[\cos (\zeta) \sinh \left(\int[\sin (\zeta)] \mathrm{d} \zeta\right)\right] \mathrm{d} \zeta\right) \mathrm{d} \zeta\right),
$$

or

$\mathbf{r}(\zeta)=\left(\zeta, \frac{A}{2} \cos (\zeta) \cosh \left(\zeta^{2}\right)-\frac{1}{2} \cos ^{2}(\zeta) \cosh \left(\zeta^{2}\right)+C \zeta+F, \frac{B}{2} \cos (\zeta) \sinh \left(\zeta^{2}\right)-\frac{1}{2} \cos ^{2}(\zeta) \sinh \left(\zeta^{2}\right)+D \zeta+G\right)$,

with constants of integration A, B, C, D, F, G. The representation of the evolves curve is in Figure 5.

Now, after performing similar calculations to the ones we performed to find the evolution equations of the curvature and torsion for the admissible curve of the first type, we found that the evolution equations for the second type correspond precisely to the admissible curve of the first type if it is timelike, as stated in the next Theorem 3.2. 


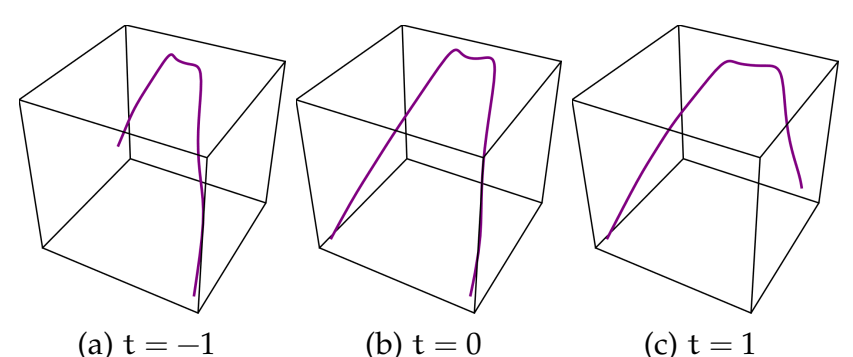

$\begin{array}{lll}\text { (a) } t=-1 & \text { (b) } t=0 & \text { (c) } t=1\end{array}$

Figure 5: With curve length $20(-10<\zeta<10)$.

Theorem 3.2 (Equations of motion for an admissible curve of second type). Let $\alpha(\zeta)=(0, \vartheta(\zeta), \rho(\zeta))$, be an admissible curve of the second type in pseudo-Galilean space $\mathrm{G}_{3}^{1}$. The evolution equations for the second type correspond exactly to the admissible curve of the first type if it is timelike. These equations are:

$$
\left\{\begin{array}{l}
\kappa_{\mathrm{t}}=\left(-\left(\frac{\kappa \Omega_{1}+\Omega_{2 \zeta}}{\tau}\right) \zeta+\tau \Omega_{2}\right), \\
\tau_{\mathrm{t}}=-\Omega_{1 \zeta} .
\end{array}\right.
$$

Remark 3.3. We note that from the previous theorem the equations of motion are the same evolution equations of the first type at $\epsilon=1$. These equations correspond to the curve $\Gamma$ being of a timelike and its principal normal $\mathbf{n}$ of a spacelike vector.

\section{Evolution equations for an admissible curve via velocity vector}

Let in pseudo-Galilean $\mathrm{G}_{3}^{1}, \Gamma(\zeta)=(\zeta, \vartheta(\zeta), \rho(\zeta))$ be an admissible curve of the first type. The relation between motion of curves and also integrable evolution in several spaces, e.g., Euclidean, Minkowski and Galilean spaces, has been studied in $[1,2,22,23,26,27] . \Gamma=\Gamma(\zeta, t)$ is assumed to be a point at time $t$ on the plane curve. The velocity fields of the traditional geometrical model, $\Gamma_{t}(\zeta)=\frac{d \Gamma}{d t}=v$, such that

$$
\Gamma_{\mathrm{t}}=\Lambda \mathbf{T}+\sigma \mathbf{n}+\Upsilon_{\mathbf{p}}
$$

where

$$
\left\{\begin{array}{l}
\Lambda=\Lambda\left(\kappa, \kappa_{s}, \ldots, \tau, \tau_{\zeta}, \ldots\right) \\
\sigma=\sigma\left(\kappa, \kappa_{\zeta}, \ldots, \tau, \tau_{\zeta}, \ldots\right) \\
\curlyvee=\Upsilon\left(\kappa, \kappa_{\zeta}, \ldots, \tau, \tau_{\zeta}, \ldots\right)
\end{array}\right.
$$

are velocity functions of $\zeta, \mathrm{t}$ corresponding to the tangent, normal, and binormal along the curve, i.e., it is intrinsic quantities. Also, $\Lambda, \sigma, \curlyvee$ are the velocities in the tangential, normal, and binormal directions. By using the Frenet frame (3.1), the dynamical equations of the tangent, normal, and binormal will be obtained within the next Theorem 4.1.

Theorem 4.1 (The dynamical equations via velocity vector). In a pseudo-Galilean space $\mathrm{G}_{3}^{1}$, the dynamical equations of the curvature and torsion of the evolving curve can be expressed in terms of velocities $\Lambda, \sigma, \curlyvee$ as

$$
\left\{\begin{array}{l}
\kappa_{\mathrm{t}}=\left(\Lambda_{\kappa}+\sigma_{\zeta}+\Upsilon_{\tau}\right)_{\zeta}+\left(\sigma \tau+\Upsilon_{\zeta}\right) \tau, \\
\tau_{\mathrm{t}}=\left[\frac{1}{\kappa}\left(\Lambda \kappa+\sigma_{\zeta}+\Upsilon_{\tau}\right) \tau+\left(\sigma \tau+\Upsilon_{\zeta}\right)\right]_{\zeta} .
\end{array}\right.
$$

Proof. The condition of compatibility is specified as follows:

$$
\Gamma_{\mathrm{t} \zeta}=\Gamma_{\zeta \mathrm{t}},
$$


by comparing coefficients of $\mathbf{T}, \mathbf{n}, \mathbf{p}$ in the above equation, one gets

$$
\left\{\begin{array}{l}
\Lambda_{\zeta}=0 \\
\epsilon \Omega_{3}=\Lambda \kappa+\sigma_{\zeta}+\Upsilon_{\tau} \\
\epsilon \Omega_{2}=\sigma \tau+\Upsilon_{\zeta}
\end{array}\right.
$$

From (3.7) and (4.3), we have:

$$
\left\{\begin{array}{l}
\epsilon \Omega_{1}=-\frac{1}{\kappa}\left[\left({ }^{\prime} \kappa+\sigma_{\zeta}+\Upsilon_{\tau}\right) \tau+\left(\sigma \tau+\Upsilon_{\zeta}\right)_{\zeta}\right], \\
\epsilon \Omega_{2}=\sigma \tau+\Upsilon_{\zeta} \\
\epsilon \Omega_{3}=\Lambda \kappa+\sigma_{\zeta}+\Upsilon_{\tau},
\end{array}\right.
$$

and the dynamical equations of the curvature and torsion of the evolving curve can be expressed in terms of velocities $\Lambda, \sigma, \Upsilon$ as

$$
\left\{\begin{array}{l}
\kappa_{\mathrm{t}}=\epsilon^{2}\left[\left(\Lambda_{\kappa}+\sigma_{\zeta}+\Upsilon_{\tau}\right)_{\zeta}+\left(\sigma \tau+\Upsilon_{\zeta}\right) \tau\right], \\
\tau_{\mathrm{t}}=\epsilon^{2}\left[\frac{1}{\kappa}\left(\Lambda_{\kappa}+\sigma_{\zeta}+\Upsilon_{\tau}\right) \tau+\left(\sigma \tau+\Upsilon_{\zeta}\right)\right]_{\zeta} .
\end{array}\right.
$$

Since, always $\epsilon^{2}=1$, then the dynamical system (4.2) is obtained.

We now consider, as an application, a model of curve evolution through local geometry using specific values for the intrinsic functions $\Lambda, \eta, \curlyvee$ without losing generality.

Model 1. Consider the admissible curve $\Gamma$ moving in the space according to

$$
\Gamma_{\mathrm{t}}=\tau \mathbf{p},
$$

where we compensated in the equation (4.1) by the values $\Lambda=\eta=0, \Upsilon=\tau$. Accordingly, the equations of evolution (4.2) are translated into

$$
\left\{\begin{array}{l}
\kappa_{\mathrm{t}}=\tau_{\zeta}^{2}+\tau_{\zeta} \tau, \\
\tau_{\mathrm{t}}=\left[\frac{1}{\kappa}\left(\tau^{3}+\tau_{\zeta \zeta}\right)\right]_{\zeta}
\end{array}\right.
$$

which have two solutions as follows.

First Solution. One of the solutions of the system of equations (4.4) is given from:

$$
\left\{\begin{array}{l}
\kappa=\frac{2 c_{2}^{3}}{c_{1}}\left(-1+3 \tanh \left(c_{1} t+c_{2} \zeta+c_{3}\right)^{2}\right), \\
\tau=-2 c_{2} \tanh \left(c_{1} t+c_{2} \zeta+c_{3}\right),
\end{array}\right.
$$

with arbitrary real constants $c_{1}, c_{2}$, and $c_{3}$, as in Figures 6,7 , and 8.

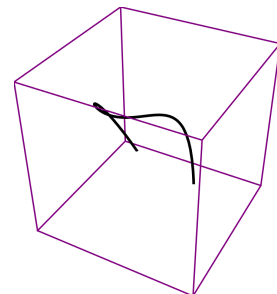

(a) $t=-1$

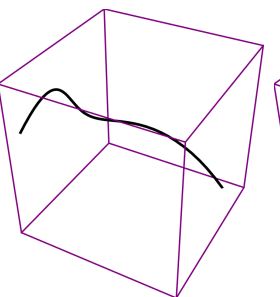

(b) $\mathrm{t}=0$

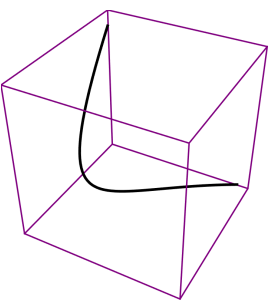

(c) $t=1$

Figure 6: With curve length 20 and constants $c_{1}=c_{2}=-2$ and $c_{3}=-1$. 


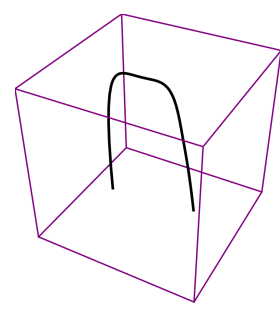

(a) $\mathrm{t}=-1$

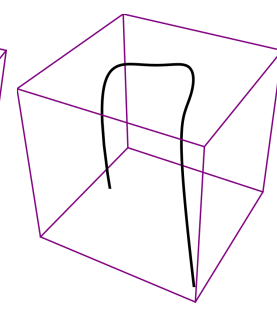

(b) $t=0$

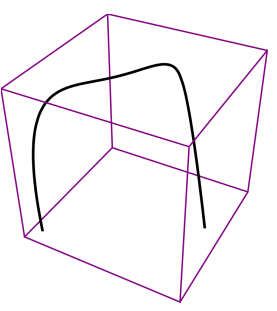

(c) $t=1$

Figure 7: With curve length 20 and constants $c_{1}=-0.2, c_{2}=-3$, and $c_{3}=0.1$.

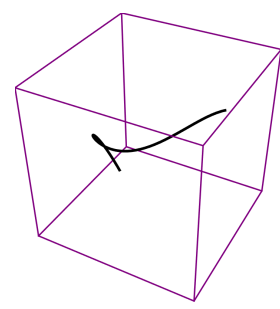

(a) $\mathrm{t}=-1$

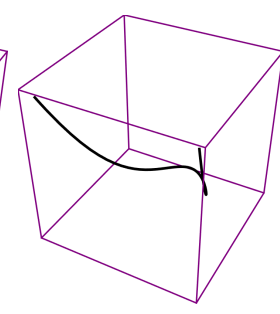

(b) $t=0$

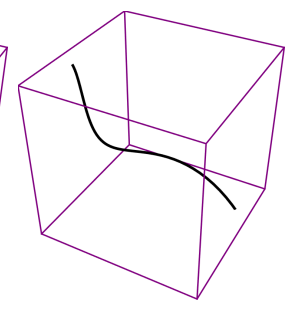

(c) $\mathrm{t}=1$

Figure 8: With curve length 20 and constants $c_{1}=c_{2}=-1$ and $c_{3}=0$.

Second solution. The second solution to the system (4.4) is written on the form:

$$
\left\{\begin{array}{l}
\kappa=\frac{2 c_{2}^{3}}{c_{1}}\left(-1+3 \tanh \left(c_{1} t+c_{2} \zeta+c_{3}\right)^{2}\right), \\
\tau=2 c_{2} \tanh \left(c_{1} t+c_{2} \zeta+c_{3}\right) .
\end{array}\right.
$$

Likewise, graphs corresponding to some specific values of the constants $c_{1}, c_{2}$, and $c_{3}$ can be seen through Figures 9, 10, and 11.

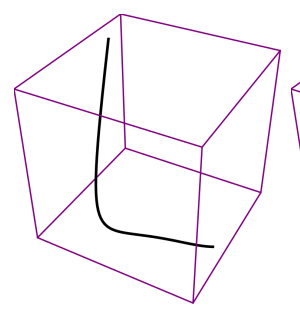

(a) $\mathrm{t}=-1$

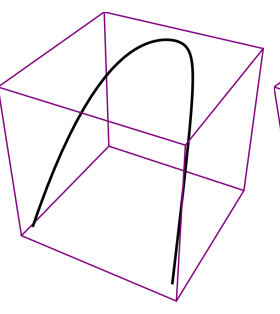

(b) $t=0$

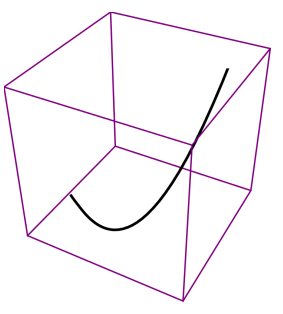

(c) $t=1$

Figure 9: With curve length 20 and constants $c_{1}=c_{2}=-1$ and $c_{3}=0$.

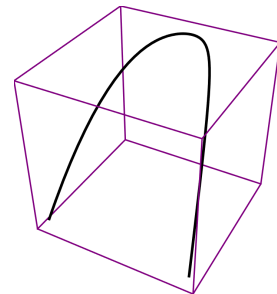

(a) $t=-1$

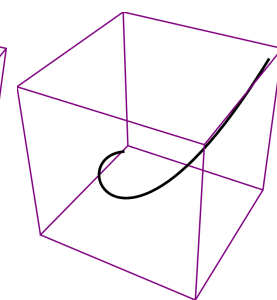

(b) $t=0$

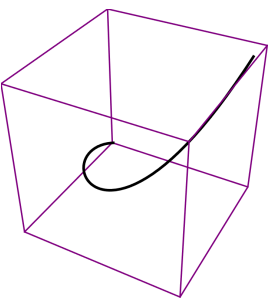

(c) $\mathrm{t}=1$

Figure 10: With curve length 20 and constants $c_{1}=c_{3}=-2$ and $c_{2}=-1$. 


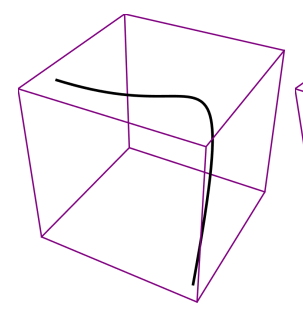

(a) $t=-1$

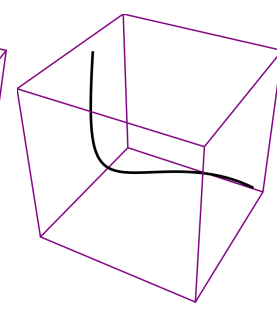

(b) $t=0$

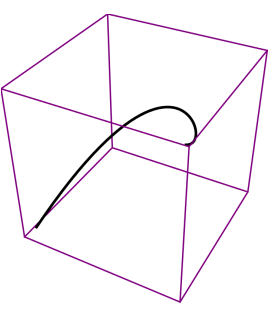

(c) $t=1$

Figure 11: With curve length 20 and constants $c_{1}=1, c_{2}=2$, and $c_{3}=3$.

\section{Comparison between evolution equations in the different spaces}

The properties of moving frames associated with curves differ from one space to another, such as the Euclidean space [2], the Minkowski space [10], the Galilean space [23] and the pseudo-Galilean space in this work. After studying the evolution equations in the pseudo-Galilean space, we found that these equations are functions in the velocities. The choice of velocities has an effect on these functions. This choice is based on space's characteristics. It is possible to choose some values of the velocities according to which the system of equations can be solved. Also, these values are not suitable for solution in another space, due to the different properties of each space. We illustrate an example by choosing values for velocities that solve equations in the Euclidean and Minkowski spaces [2], but they are not suitable for solving in the pseudo-Galilean space, which indicates that the equations of motion depend on velocities and these velocities differ in each space. We tend to conclude that the equations of motion have the following form:

- first, the evolution via Frenet frame:

$$
\kappa_{t}=f\left(\left(\Omega_{1}, \Omega_{2}, \Omega_{3}\right), \quad \tau_{t}=g\left(\Omega_{1}, \Omega_{2}, \Omega_{3}\right) ;\right.
$$

- second, the evolution via velocity vector field:

$$
\kappa_{\mathrm{t}}=\mathrm{h}(\Lambda, \eta, \Upsilon), \quad \tau_{\mathrm{t}}=w(\Lambda, \eta, \Upsilon),
$$

where $f, g, h$, and $w$ have different values in each space. This means the equations of motion depend on the velocities which differ according to each space.

\section{Conclusion}

The evolution equations for curvature and torsion for the admissible curves were obtained in the three dimensional pseudo-Galileo space $\mathrm{G}_{3}^{1}$. This was accomplished by applying compatibility conditions using the curve Frenet frame differentials for arc curve length parameter and time. Then we solved the resulting system of coupled nonlinear partial differential equations numerically using the Mathematica software program. As an important result, we observed that all evolution equations could be obtained by researchers in Euclidean and Minkowski 3-dimensional spaces via frame fields or via the velocity vector are consistent with our study in the pseudo-Galilean space $\mathrm{G}_{3}^{1}$. In these studies, they all depend on their velocities, but velocities do not have to be the same. For example, velocities values can be chosen in one of the spaces to provide a solution to the system of evolution equations that cannot be provided in another. Also, we provided some models as application for the evolution equations for admissible curves and get the solutions for these new equations. A natural extension of this work is to generate surfaces from the given evolving curves and to study the evolution equations of surfaces in the pseudo-Galilean space $\mathrm{G}_{3}^{1}$. 


\section{References}

[1] N. H. Abdel-All, M. A. Abdel-Razek, H. S. Abdel-Aziz, A. A.Khalil, Geometry of evolving plane curves problem via lie group analysis, Stud. Math. Sci., 2 (2011), 51-62. 1, 4

[2] N. H. Abdel-All, R. A. Hussien, T. Youssef, Evolution of curves via the velocities of the moving frame, J. Math. Comput. Sci., 2 (2012), 1170-1185. 1, 4, 5

[3] N. H. Abdel-All, S. G. Mohamed, M. T. Al-Dossary, Evolution of generalized space curve as a function of its local geometry, Appl. Math., 5 (2014), 2381-2392. 1

[4] K. Alkan, S. C. Anco, Integrable systems from inelastic curve flows in 2and 3dimensional Minkowski space, J. Nonlinear Math. Phys., 23 (2016), 256-299. 1

[5] R. Balakrishnan, R. Blumenfeld, Transformation of general curve evolution to a modified Belavin-Polyakov equation, J. Math. Phys., 38 (1997), 5878-5888. 1

[6] D. Baldwin, Ü. Göktaş, W. Hereman, L. Hong, R. S. Martino, J. C. Miller, Symbolic computation of exact solutions expressible in hyperbolic and elliptic functions for nonlinear PDEs, J. Symbolic Comput., 37 (2004), 669-705. 1

[7] S. Cengiz, E. B. Koc Ozturk, U. Ozturk, Motions of curves in the pseudo-Galilean space $\mathbb{G}_{3}^{1}$, Math. Probl. Eng., 2015 (2015), 6 pages. 2.1, 2.1

[8] M. Dede, C. Ekici, On parallel ruled surfaces in Galilean space, Kragujevac J. Math., 40 (2016), 47-59. 2

[9] M. Desbrun, M.-P. Cani, Active implicit surface for animation, In: Proc. Graphics Interface-Canadian Inf. Process. Soc., (1998), 143-150. 1

[10] Q. Ding, W. Wang, Y. Wang, A motion of spacelike curves in the Minkowski 3-space and the KdV equation, Phys. Lett. A, 374 (2010), 3201-3205. 5

[11] B. Divjak, Geometrija pseudogalilejevih prostora, Ph.D. Thesis, University of Zagreb, (1997). 2

[12] B. Divjak, Curves in pseudo-Galilean geometry, Ann. Univ. Sci. Budapest. Eötvös Sect. Math., 41 (1998), 119-130. 2, 2.1, 2.1

[13] B. Divjak, Ž. Milin-Šipuš, Special curves on ruled surfaces in Galilean and pseudo-Galilean spaces, Acta Math. Hungar., 98 (2003), 203-215. 2, 2.1

[14] B. Divjak, Ž. Milin-Šipuš, Minding isometries of ruled surfaces in pseudo-Galilean space, J. Geom., 77 (2003), $35-47$.

[15] C. Ekici, M. Dede, On the Darboux vector of ruled surfaces in pseudo-Galilean space, Math. Comput. Appl., 16 (2011), 830-838. 2

[16] A. S. Fokas, J. Lenells, A new approach to integrable evolution equations on the circle, Proc. A., 477 (2021), 28 pages. 1

[17] M. Gage, R. S. Hamilton, The heat equation shrinking convex plane curves, J. Differential Geom., 23 (1986), 69-96. 1

[18] E. F. D. Goufo, I. T. Toudjeu, Analysis of recent fractional evolution equations and applications, Chaos Solitons Fractals, 126 (2019), 337-350. 1

[19] M. A. Grayson, The heat equation shrinks embedded plane curves to round points, J. Differential Geom., 26 (1987), 285-314. 1

[20] N. Gürbüz, Three classes of non-lightlike curve evolution according to Darboux frame and geometric phase, Int. J. Geom. Methods Mod. Phys., 15 (2018), 16 pages. 1

[21] H. Hasimoto, A soliton on a vortex filament, J. Fluid Mech., 51 (1972), 477-485. 1

[22] M. Hisakado, M. Wadati, Moving discrete curve and geometric phase, Phys. Lett. A, 214 (1996), 252-258. 4

[23] Z. Kucukarslan-Yuzbasi, E. Cavlak-Aslan, M. Inc, D. Baleanu, On exact solutions for new coupled nonlinear models getting evolution of curves in Galilean space, Therm. Sci., 23 (2019), 227-233. 4, 5

[24] G. L. Lamb Jr, Solitons on moving space curves, J. Mathematical Phys., 18 (1977), 1654-1661. 1

[25] H. Q. Lu, J.S. Todhunter, T. W. Sze, Congruence conditions for nonplanar developable surfaces and their application to surface recognition, CVGIP: Image Understanding, 58 (1993), 265-285. 1

[26] K. Nakayama, Motion of curves in hyperboloid in the Minkowski space, J. Phys. Soc. Japan, 67 (1998), 3031-3037. 4

[27] K. Nakayama, H. Segur, M. Wadati, Integrability and the motion of curves, Phys. Rev. Lett., 69 (1992), 2603-2606. 4

[28] H. Öztekin, H. G. Bozok, POSITION VECTORS OF ADMISSIBLE CURVES IN 3-DIMENSIONAL PSEUDOGALILEAN SPACE G ${ }_{3}^{1}$, Int. Electron. J. Geom., 8 (2015), 21-32. 3, 3, 3

[29] A. I. Prilepko, A. B. Kostin, I. V. Tikhonov, Inverse problems for evolution equations, De Gruyter, (2020), 379-389. 1

[30] A. Gray, Modern Differential Geometry of Curves and Surfaces with Mathematica, CRC Press, Boca Raton, (1998). 3

[31] J. A. Sethian, Level set methods and fast marching methods: Evolving interfaces in computational geometry, fluid mechanics, computer vision, and materials science, Cambridge university press, (1999). 1

[32] D. J. Unger, Developable surfaces in elastoplastic fracture mechanics, Int. J. Fract., 50 (1991), 33-38.

[33] I. Waini, A. Ishak, I. Pop, Unsteady flow and heat transfer past a stretching/shrinking sheet in a hybrid nanofluid, Int. J. Heat Mass Transf., 136 (2019), 288-297.

[34] F. Yang, Sun, Y.-R. Li, H. X.-X. Li, C.-Y. Huang, The quasi-boundary value method for identifying the initial value of heat equation on a columnar symmetric domain, Numer. Algorithms, 82 (2019), 623-639. 1

[35] Ö. G. Yildız, M. Tosun, A note on evolution of curves in the Minkowski spaces, Adv. Appl. Clifford Algebr., 27 (2017), 2873-2884. 1 